\title{
Comunicação no ensino médico: estruturação, experiência e desafios em novos currículos médicos
}

\author{
Communication skills in medical teaching: \\ structure, experience and challenges in new \\ medical curricula
}

\author{
Barbara Turini ${ }^{1}$ \\ Daniel Martins Neto ${ }^{1}$ \\ Marcelo de Sousa Tavares ${ }^{1}$ \\ Sandra Odebrecht Vargas Nunes ${ }^{1}$ \\ Vera Lucia Menezes da Silva ${ }^{1}$ \\ Zuleika Thomson ${ }^{1}$
}

\section{PALAVRAS-CHAVE \\ - Comunicação. \\ - Educação de Graduação em Medicina. \\ - Cuidado Centrado no Paciente. \\ - Educação Médica.}

Recebido em: 10/11/2007

Reencaminhado em: 29/01/2008 Aprovado em: 14/02/2008
REVISTA BRASILEIRA DE EDUCAČ̃̃ MÉDICA $26432(2): 264-270: 2008$

\footnotetext{
${ }^{1}$ Universidade Estadual de Londrina, Paraná, Brasil.
}

\begin{abstract}
A B S T R A C T
This article relates an experience of a group of teachers of the Integrated Medical Course of the State University of Londrina in teaching communication in the Skills Module. The awareness of the importance of communication skills not only in the doctor-patient relationship but also in the evolution of diseases led to the design of a module with progressive difficulties throughout the first years of the course. The content of the module ranges from observation of patients in the waiting room to discussions on how to transmit information, treatment adherence and management of special groups of patients in different stages of life. The methods used include observation of patients, interviews, group discussions, role-playing, films, round tables, conferences and statements of patients. The evaluation occurs in two parts: formative and cognitive. The training of communication skills is aimed at developing the ability of the student to establish a good doctor-patient relationship, history taking and transmission of information and at promoting treatment adherence.
\end{abstract}




\section{INTRODUÇÃO}

O curso de Medicina da Universidade Estadual de Londrina (UEL) aprovou em 1997 uma nova estrutura curricular, com mudanças significativas não só nas metodologias de ensino-aprendizagem, mas também nos conteúdos. Entre os novos conteúdos curriculares, consta o estudo da comunicação no contexto da prática médica, com objetivo na relação médico-paciente segundo o modelo de Balint ${ }^{1}$. Este modelo preconiza que se deve ter em mente que o remédio mais utilizado na medicina é o próprio médico, o qual, como os demais medicamentos, precisa ser conhecido quanto a sua posologia, reações colaterais e toxicidade.

O currículo inicialmente foi fundamentado no modelo da Universidade de Maastricht, utilizando o método da aprendizagem baseada em problemas, aprendizagem autodirigida, adoção de novas formas de avaliação e natureza multidisciplinar². O Programa de Capacitação para Habilidades em Comunicação na Escola Médica de Maastricht é desenvolvido por um grupo multiprofissional com foco na comunicação médico-paciente durante os quatro anos pré-clínicos. Os estudantes são distribuídos em grupos de dez alunos a cada duas semanas. Em cada sessão são desenvolvidas práticas de consulta em contato com pacientes simulados. Aavaliação é realizada no contexto do Exame Objetivo Clínico Estruturado (Osce) ${ }^{3}$.

Entre os 19 tópicos de ética preconizados na formação médica segundo Roberts ${ }^{4}$, o programa de Habilidades em Comunicação da UEL inclui os aspectos que se referem à relação médico-paciente, relação médico e família, sigilo e confidencialidade nos registros médicos.

O processo de formação em habilidades de comunicação é fundamentado em três premissas básicas: primeiro, que boas habilidades de comunicação durante uma consulta médica estão relacionadas de forma importante aos resultados, tanto para pacientes como para médicos; segundo, que a habilidade de comunicação nem sempre é adequada e que, portanto, alguns resultados para estes médicos e pacientes podem ser melhorados; terceiro, que a habilidade de comunicação pode ser ensinada ${ }^{5}$.

Este artigo apresenta uma reflexão sobre a comunicação no exercício da prática médica, seguida pelo relato da experiência vivenciada no curso de Medicina da UEL com a implantação deste tema no contexto do currículo integrado.

\section{A COMUNICAÇÃO NA TRANSIÇÃO PARADIGMÁTICA DA SAÚDE}

Os problemas que caracterizam a insatisfação dos pacientes com a prática médica tradicional ocorrem até mesmo em países considerados desenvolvidos. As queixas de pacientes variam desde o baixo envolvimento de clínicos gerais com os pacientes fora de seu expediente até a falta de triagem de rotina para certas doenças, entre outras ${ }^{6}$. Recente estudo revela a dificuldade que pacientes encontram, no momento da consulta médica, para expressar todas as suas queixas e demandas, tais como preocupações sobre possíveis diagnósticos e conseqüências, efeitos colaterais de tratamentos e informação relacionada ao contexto social da doença, e que podem interferir significativamente no desfecho da intervenção clínica?

As insatisfações dos pacientes criadas por dificuldades de comunicação durante o atendimento médico podem gerar processos judiciais, por questões não somente objetivas (como sobrevida, óbito ou seqüela), mas também subjetivas, como sensação de abandono do paciente, desvalorização das visões do paciente e/ou família sobre o problema e incapacidade de compreender a perspectiva do paciente e sua família ${ }^{8}$. Entre as questões apontadas atualmente por pacientes como qualidades priorizadas em médicos constam "humanidade", seguida por "competência/acurácia do tratamento", "envolvimento dos pacientes em decisões clínicas" e "tempo para seu cuidado".

Um estudo canadense sobre o envolvimento de pacientes na tomada de decisão sobre seu tratamento revela que estes mostram desejo de informação, e poucos desejam que a tomada de decisão caiba exclusivamente ao médico. Há a sugestão de que médicos exerçam dois papéis principais: assistir os pacientes na resolução de problemas de modo a estruturar escolhas possíveis e apoiá-los na tomada de decisões difíceis ${ }^{10}$.

Diante dessas novas demandas para a prática médica, conceitos como percepção do binômio saúde-doença e qualidade de vida, entre outros, aproximam-se cada vez mais da perspectiva do paciente. Visões teoricamente subjetivas dependem das sensações que o relacionamento médico-paciente proporciona ${ }^{11,12}$.

A postura positivista da visão objetiva da doença está sendo substituída por uma "nova medicina subjetiva", segundo as palavras de Sullivan ${ }^{13}$, que incorpora o que o paciente pensa e sente, propõe sua autonomia sobre escolha de condutas que impactam sua saúde e progressivamente visa ao "cuidar" mais que ao "curar". Aspecto semelhante é abordado por Nascimento ${ }^{14}$ ao considerar a necessidade de que os hospitais tenham suporte aos profissionais cuidadores. Assim, o espaço de relação entre o profissional de saúde e o usuário passa a ser considerado determinante para o êxito das ações de saúde desenvolvidas. Ayres ${ }^{15}$ descreve o momento da interação como uma relação entre sujeitos, para a construção conjunta do projeto de intervenção sobre a saúde da pessoa, considerando seus valores. 
Merhy ${ }^{16}$ diferencia três tipos de tecnologias necessárias à prática médica: "tecnologias duras", constituídas basicamente pelos equipamentos que o médico utiliza (estetoscópio, endoscópio, etc.); "tecnologias leves-duras", compostas basicamente por saberes bem estruturados (como a clínica ou a epidemiologia); e, finalmente, localizadas no espaço relacional trabalhador-usuário, o autor cita a existência de "tecnologias leves implicadas com a produção das relações entre dois sujeitos, que só têm materialidade em ato".

Portanto, a interação médico-paciente é atualmente entendida como atividade fundamental do trabalho médico e que necessita de tecnologias específicas devido a sua alta complexidade. Para atender essas novas visões sobre o trabalho médico, tem sido estudada a comunicação durante a prática médica e ministrados cursos de treinamento, especialmente na área pediátrica ${ }^{17}$.

Em estudo de revisão de literatura, Ong et al. ${ }^{18}$ relatam que a comunicação médico-paciente pode trazer diferentes conseqüências para o paciente, como satisfação, aderência ou não ao tratamento e compreensão ou não da informação transmitida. Os autores identificam diferentes propósitos para a comunicação: criação de uma boa relação interpessoal, troca de informações e tomada de decisões relacionadas com o tratamento. Também descrevem diferentes sistemas desenvolvidos para a análise do processo de comunicação médico-paciente (ou sistemas de análise de interação), constituídos basicamente por instrumentos de observação de um encontro médico. Embora pudessem ser identificados dois tipos de sistemas, um instrumental (voltado à cura) e outro afetivo (dirigido ao cuidado), ambos propõem como principais aspectos a serem analisados: a comunicação verbal e não verbal, a privacidade, o comportamento controlador ou não e o vocabulário predominantemente médico ou cotidiano.

O modelo para o ensino da comunicação entre médico e paciente desenvolvido na escola médica de Maastricht ${ }^{3}$ vem sendo modificado progressivamente a partir da literatura e da experiência de seus docentes. Este modelo considera três fases durante a consulta médica: na primeira, o paciente é o "entendido", pois sabe o motivo da consulta, suas queixas, ansiedades e preocupações. Nessa fase, o médico deve ouvir, explorar e ser receptivo aos aspectos trazidos pelo paciente. Na segunda fase, os papéis se invertem: o médico direciona o interrogatório para o seu raciocínio clínico, incluindo a revisão sistematizada sobre os diversos aparelhos e o exame físico. Nessa fase, embora o médico seja o detentor da informação, a clareza das instruções e o comportamento sensível e cortês são considerados importantes, pois, com freqüência, o paciente se encontra em situação vulnerável. Na terceira fase, ambos, médico e paciente, são os entendidos, cada um em sua área. $\mathrm{O}$ médico sabe o que pode ser feito, incluindo alternativas potenciais. É sua responsabilidade esclarecer essas alternativas e prováveis conseqüências. Entretanto, cabe ao paciente julgar essas alternativas, perante suas circunstâncias particulares, possibilidades e preferências. Portanto, o processo pode ser considerado uma "negociação", proporcionando ao paciente discutir com o médico suas possibilidades de adesão à conduta proposta.

Foi baseado neste modelo que um grupo de professores da UEL se reuniu para criar e implantar a proposta de ensino da comunicação.

\section{O PROCESSO DE IMPLANTAÇÃO DO ENSINO DA COMUNICAÇÃO NA UEL}

A partir de meados da década de 1990, entre as novas propostas de organização de um currículo integrado de Medicina estava incluído o ensino da comunicação. Este faria parte dos módulos de habilidades clínicas e atitudes desenvolvidos ao longo dos quatro primeiros anos do curso, mas que contemplam também procedimentos laboratoriais, médico-cirúrgicos e semiologia ${ }^{19,20}$.

Considerando que uma das atribuições das escolas médicas é o treinamento na construção de relacionamentos terapêuticos e que a qualidade da relação médico-paciente afeta a saúde e a recuperação de doenças crônicas pela compreensão da doença e redução da ansiedade ${ }^{21}$, o grupo inicial de professores responsáveis pela construção dos módulos do currículo integrado optou por treinar o estudante para exercer a medicina com ênfase na relação médico-paciente. Foram estabelecidos Módulos de Habilidades Clínicas e Atitudes, incluindo treinamento em comunicação do primeiro ao quarto ano.

As Habilidades de Comunicação do primeiro ao quarto ano têm como objetivos: capacitar o aluno para praticar escuta ativa; ter empatia; utilizar uma linguagem acessível; aprimorar a comunicação não verbal; iniciar, manter e fechar a entrevista; fornecer informações sobre o diagnóstico e tratamento para pacientes, familiares e comunidade; analisar as reações do doente frente à doença e suas próprias reações; treinar a comunicação em grupos com limitações 22,23 .

Neste contexto, o ensino da comunicação desde o princípio foi desenvolvido em grupos de oito a dez alunos com um instrutor. As atividades têm seguido os princípios da metodologia ativa de ensino-aprendizagem, incluindo diversas atividades práticas dos estudantes, como observação de sala de espera em ambulatório com entrevista dos pacientes e/ou acompanhantes, observação e análise crítica de consultas médicas, entrevista com voluntários ou pacientes reais e observação crítica interpares de atitudes relacionadas à comunicação. 
Um pequeno grupo de instrutores, desde as primeiras atividades, foi responsável pela estruturação, desenvolvimento e avaliação da comunicação. A caracterização desse grupo inclui principalmente a boa vontade de seus integrantes, ao lado da multiprofissionalidade, incluindo enfermeiras, assistentes sociais, psicólogas e médicos. Esses profissionais provêm de diversos setores do Hospital Universitário (HU), do Ambulatório do Hospital de Clínicas (AHC) e dos Centros de Ciências da Saúde (CCS) e Ciências Biológicas (CCB) ${ }^{24}$.

Os materiais desenvolvidos por esse grupo incluíram não somente manuais para estudantes e roteiros de aulas, como também vídeos com consultas médicas simuladas pelo grupo de teatro da UEL, entrevistas com pacientes portadores de agravos crônicos e seu impacto sobre sua qualidade de vida, e pacientes portadores de dificuldades de comunicação, bem como cenas de filmes que representam situações de relação médico-paciente consideradas interessantes para análise e discussão com os estudantes.

Os temas abordados ao longo dos primeiros quatro anos incluíam formas de comunicação (verbal e não verbal), conhecimento e entendimento das principais características e emoções nas diferentes fases do ciclo vital, reflexão junto aos instrutores sobre as emoções do próprio aluno, limitações à qualidade de vida causadas por diferentes agravos, comunicação de "más notícias" e reflexão sobre a finitude.

Algumas orientações são comuns a todas as séries, como um ambiente adequado para obter a informação, tão confortável quanto possível para o paciente e o entrevistador, proporcionando privacidade e minimizando distrações externas ${ }^{25}$. Uma relação de confiança com o paciente deve ser estabelecida por meio de acolhimento, apresentação do entrevistador, saudações apropriadas, explicação do propósito da entrevista e garantia de sigilo ${ }^{25}$.

No desenvolvimento da entrevista, enfatiza-se a escuta ativa, bem como a eliminação de barreiras lingüísticas, entendimento da história de vida do paciente ${ }^{25}$, uso de linguagem acessível e preocupação com a comunicação não verbal, como contato visual, voz (altura, entonação e ritmo), postura e sorriso ocasional. Na comunicação verbal, discute-se sobre perguntas abertas e fechadas, indutoras ou não, clarificação das expectativas do paciente sobre a consulta, ouvir sem interromper, além de evitar fazer perguntas em tom de acusação e formular várias perguntas ao mesmo tempo.

No encerramento da entrevista, orienta-se a checar a compreensão do paciente e o que ele sabe sobre sua doença, parafrasear, repetir frases ditas pelo paciente, refletir sentimentos, resumir e se despedir. O estudante deve trabalhar o término da entrevista com o paciente de um modo que promova uma sensação de cooperação e clareza dos objetivos e progressos esperados ${ }^{25}$.
Assim, os objetivos das atividades de comunicação variam nos quatro módulos de Habilidades e Atitudes do curso, que procuram desenvolver essas habilidades da seguinte forma ${ }^{19,20}$ :

$1{ }^{a}$ série: Capacitar os estudantes para a escuta ativa; estabelecer uma relação de empatia; usar linguagem acessível; desenvolver adequadamente a comunicação não verbal; iniciar, manter e fechar a entrevista; cuidar da privacidade quando em contato com o paciente; identificar variáveis que favoreçam lidar com as reações do paciente frente à doença e com as suas próprias reações.

2-a série: Capacitar os estudantes para entrevistar indivíduos em diferentes fases do ciclo vital: gestante, mãe-filho, adolescente, adulto e idoso, analisando seu contexto social, cultural e familiar, reconhecendo as características biopsicossociais dessas fases da vida.

3a série: Capacitar os estudantes para a abordagem de pacientes de difícil manejo, bem como orientar seus familiares, promover a adesão ao tratamento e a modificação do estilo de vida, e avaliar o impacto da doença na qualidade de vida.

4ª série: Capacitar os estudantes para informar diagnósticos, planos de tratamento e prognósticos; identificar variáveis indicadoras de maus-tratos; abordar familiares para doação de órgãos; comunicar "más notícias"; conduzir o paciente e familiar em situações difíceis.

\section{AS ESTRATÉGIAS DE ENSINO}

As estratégias usadas para desenvolver esses módulos de comunicação ao longo dos quatro anos variam de acordo com o tema e com o número de estudantes. Estes podem ser divididos em duplas ou em grupos de 10, 40 ou 80 para desenvolver as atividades. As estratégias utilizadas são:

- Observação: Os estudantes observam, individualmente ou em duplas, a relação paciente-equipe de saúde em sala de espera, em consulta médica, em comunicação de diagnóstico, em orientação de tratamento.

- Entrevistas: Por meio de roteiro, os estudantes entrevistam pacientes, em diferentes fases da vida e condições de saúde, e profissionais, objetivando modelos de relacionamento médico-paciente. São enfocados: acolhimento, habilidades não verbais (voz modulada, expressão facial, olhar nos olhos dos pacientes e familiares, sorriso ocasional, postura corporal, linguagem acessível, expressão de empatia), habilidades de perguntar (evitar perguntas indutoras, balanço entre perguntas abertas e fechadas, obter dados pessoais e familiares, bem como diagnóstico abrangente), garantir o sigilo da entrevista, 
checar a compreensão do paciente, operacionalizar as informações e refletir os sentimentos. As entrevistas são acompanhadas de relatórios, sendo fornecida realimentação (feedback) acerca das habilidades alcançadas.

- Discussões em grupo: Todas as atividades realizadas são registradas em relatórios e discutidas em pequenos grupos, sendo relacionadas com leituras pertinentes ao assunto.

- Dramatizações: Situações de relação médico-paciente-como entrevistas no ciclo evolutivo, adesão ao tratamento ou transmissão de más notícias - são dramatizadas com os pares ou pacientes simulados, visando identificar dificuldades e capacitar para atitudes mais adequadas em diversas situações clínicas planejadas. Essas situações são seguidas de discussão em pequenos grupos.

- Filmes: São utilizados clipes de filmes ("Melhor impossível", "Mister Jones", "Patch Adams", "Golpe do destino", "Hit" e outros) com cenas que abordam a relação médico-paciente, para motivar a discussão.

- Mesas-redondas e conferências: Os temas debatidos por diferentes profissionais com o objetivo de discutir modelos de atitude ou conduta incluem a relação médico-paciente no ciclo vital, cuidados com crianças submetidas a maus-tratos, relação com o paciente terminal e doação de órgãos, entre outros.

- Depoimentos de pacientes: Pacientes que vivenciaram agravos que mudaram significativamente sua vida discutem com grupos de estudantes o processo de transmissão da notícia e como sentiram e perceberam a atitude dos profissionais de saúde.

Os estudantes são avaliados de duas formas:

- Avaliação formativa: Ocorre individualmente a cada encontro por meio de uma ficha que pontua o cumprimento de tarefas (entrevistas e relatórios, entre outras), leituras e apresentação de resumos, relação entre teoria (leituras) e prática (tarefas), pontualidade e assiduidade.

- Avaliação cognitiva: Por meio de Exame Objetivo Clínico Estruturado (Osce), realizado no fim do primeiro e segundo semestre. $\mathrm{O}$ processo de comunicação em geral é avaliado pela observação da relação do estudante com um paciente ou familiar em determinada situação clínica. Para isso, participam voluntários treinados para representar o usuário na situação clínica proposta e professores munidos com um checklist das atitudes consideradas fundamentais ao estabelecimento de uma boa relação com o usuário na condição simulada.

\section{DIFICULDADES ENCONTRADAS}

O ensino de habilidades em comunicação não era incluído no currículo tradicional e no modelo integrado é uma das inovações que foi desenvolvida e adaptada às peculiaridades da UEL, encontrando-se em constante processo de avaliação para readequações. Durante este processo, foram percebidas diversas dificuldades, como, por exemplo:

- Modelo de ensino: A importância da comunicação para o desenvolvimento de uma relação médico-paciente mais adequada já está bem estabelecida, mas existem poucas referências sobre a estratégia pedagógica que se deve empregar para atingir este objetivo. O problema mais imediato decorrente desta ausência de modelo prévio (anterior) foi a falta de um fio condutor que guiasse a estruturação das atividades de forma seqüencial, do básico para o avançado, do simples para o complexo, numa evolução progressiva que permeasse os quatro primeiros anos do curso. No currículo integrado, diferentemente das avaliações tradicionais, orientadas para a observação da aquisição de conteúdos (cognitiva), avaliam-se também os objetivos afetivos e atitudinais.

- Docentes: Até 1997, os professores de Medicina da UEL exerciam um papel tradicional, sendo o professor a única fonte de conhecimento, e sua atuação ficava restrita a preparar e dar aulas ou palestras e elaborar provas de caráter cognitivo. Durante a implantação do currículo integrado, grande parte do corpo docente não foi motivada para participar de atividades inovadoras, e poucos estavam qualificados para a orientação de habilidades de comunicação. Mesmo os docentes que participaram de cursos e treinamentos se sentiam inseguros para abordar o tema. Outras dificuldades percebidas foram falta de comprometimento e adesão apenas parcial de vários docentes. Acredita-se que uma das causas tenha sido a resistência ou oposição desse grupo à adoção de novas estratégias educacionais para o curso médico. O reflexo desta circunstância nas atividades de comunicação é que, até hoje, um reduzido número de docentes participa das habilidades de comunicação. Uma das fragilidades é que, quando alguém do pequeno núcleo de participantes tem alguma impossibilidade para o trabalho, essa ausência causa grande impacto no desenvolvimento das atividades, dificultando discussões e reflexões em pequenos grupos. A adesão de novos docentes ao núcleo de habilidades de comunicação é tímida. Um dos prováveis motivos desta resistência seria o preconceito em participar de uma atividade pouco valorizada pelo médico e considerada uma atribuição não médica, relacionada ao senso comum. 
- Estudantes: Para os estudantes, a atividade de ensino mais importante da semana é o grupo tutorial. As habilidades, especialmente de comunicação, são menos consideradas, o que se constata a partir da pouca participação nas discussões e cumprimento de trabalhos acadêmicos solicitados, independentemente de serem objeto de avaliação. Por outro lado, a equipe de comunicação tem observado que esse aluno desenvolve uma postura mais crítica sobre os comportamentos médicos mais adequados e também demonstra alguma receptividade para praticar um modelo mais humanizado de comunicação médica em sua atuação como futuro profissional.

- Instituição/estrutura física: A adoção de novos rumos para o curso de Medicina com novas estratégias educacionais necessita de estrutura física adequada, como salas para pequenos grupos. A maioria dos financiamentos disponibilizados não possibilita ampliações de estrutura física, o que dificulta o desenvolvimento das atividades e a introdução de novas técnicas, como, por exemplo, sala de espelho.

- Integração com outros setores: As habilidades são divididas em quatro grandes setores: Semiologia, Comunicação, Procedimentos e Laboratório. Embora devesse haver forte integração entre a Semiologia e a Comunicação, isto não tem ocorrido. Também não existe uma correlação direta entre as atividades de habilidades de comunicação e os módulos temáticos, por vezes ocorrendo atividades inteiramente diversas do conteúdo do módulo.

- Internato médico: Não há continuidade do programa de comunicação durante o internato, é durante esta fase que ocorre a maior parte do treinamento prático do estudante de $\mathrm{Me}-$ dicina. $\mathrm{O}$ interno espelha seu comportamento nas posturas dos demais membros das equipes, convivendo com docentes e demais médicos do HU formados em cursos tradicionais, os quais não consideram a comunicação na prática médica. Isso leva os estudantes a reduzir a valorização dos assuntos relacionados a esse tema e que foram abordados nos módulos de habilidades nos anos anteriores.

- Carga horária: Para a Comunicação estão destinados 25\% da carga horária dos módulos de habilidades para cada série. Essa pequena carga horária não permite a execução de atividades mais elaboradas e muitas vezes impede a seqüência dos temas desenvolvidos.

- Treinamento de pacientes simulados: Nas avaliações do tipo Osce, voluntários treinados devem encenar situações de interação médico-paciente e simular reações predefinidas durante a relação com o estudante. Uma das dificuldades reside em padronizar a conduta dos diferentes atores, pois, apesar dos roteiros estabelecidos e dos treinamentos realizados, as encenações variam conforme as características de cada voluntário e da própria interação, dificultando a equivalência das condições de avaliação dos estudantes.

\section{CONSIDERAÇÕES FINAIS}

Embora já tenham decorrido dez anos desde a implantação do currículo integrado na UEL, ainda não há uma avaliação consistente quanto a seu impacto sobre a prática profissional do médico assim formado. Até o momento, cinco turmas de cerca de 80 alunos finalizaram o curso neste modelo e muitos ainda estão cursando residências médicas ou especializações.

Existem relatos informais sobre mudança de postura dos médicos recém-formados pela UEL quando inseridos no mercado de trabalho, em especial nas equipes de saúde de Londrina e região. Também há referências verbais e observações informais de que há maior facilidade de candidatos oriundos de cursos de Medicina com currículos integrados em avaliações práticas do tipo Osce. Estas vêm sendo incluídas em concursos para admissão a residências médicas e muitas vezes também abordam a comunicação. Essas observações ainda deverão se tornar objeto de pesquisa, para a comprovação da eficácia real do método.

Um aspecto positivo da experiência vivenciada na UEL foi a formação de um núcleo de docentes bastante coeso, porém muito pequeno, que acredita firmemente que a exposição do aluno às atividades de habilidades de comunicação desde o primeiro ano do curso médico pode promover uma mudança de postura e percepção crítica do papel do médico; que o estudante se torna capaz de diferenciar os comportamentos médicos adequados e inadequados; que aprende a necessidade de usar linguajar próprio ao meio sociocultural dos pacientes e promover adesão ao tratamento; e, ainda, que aprende a lidar com situações mais complexas no relacionamento com o paciente, como no caso de dar "más notícias".

Foi possível elaborar uma estrutura evolutiva para atitudes em comunicação, utilizada durante o programa de habilidades em comunicação, que, embora necessite de correções, já atende a alguns dos objetivos traçados e deverá, aos poucos, ser aperfeiçoada.

O treinamento da comunicação com ênfase na relação médico-paciente visa desenvolver a competência do aluno em aprimorar seu vínculo com o paciente, potencializar os processos de obtenção e transmissão de informações, viabilizar o manejo do paciente de forma respeitosa e promover a adesão ao tratamento. 


\section{REFERÊNCIAS}

1. Balint M. O médico seu paciente e a doença. Rio de Janeiro: Atheneu; 1984.

2. Moust J, Van Berkel H, Schmidt, H. Signs of erosion: Reflections on three decades of problem-based-learning at Maastricht University. Higher Education 2005; 50: 665-683.

3. Van Dalen J, Bartholomeus P, Kerkhofs, E, Lulofs R, Van Thiel J, Rethans JJ, et al. Teaching and assessing communication skills in Maastricht: the first twenty years. Med Teach 2001; 23:245-51

4. Roberts L, Warner T, Hammond T, Geppert C, Heirich T Becoming a good doctor; perceived need for ethics training focused on practical and professional development topics. Acad Psychiatry. 2005; 29 (3):301-8.

5. Brown RF, Bylund CL. Communicatrion skills training: describing a new conceptual model. Acad Med 2008; 83:37-44.

6. Wensing M, Mainz J, Ferreira P, Hearnshaw H, Hjortdahl $\mathrm{P}$, Olesen F, et al. General practice care and patients' priorities in Europe: an international comparison. Health Policy 1998; 45(3):175-86.

7. Barry CA, Bradley CP, Britten N, Stevenson FA, Barber N. Patients' unvoiced agendas in general practice consultations: a qualitative study. Br Med J 2000; 320:1246-50.

8. Beckman HB, Markakis KM, Suchman AL, Frankel RM. The doctor-patient relationship and malpractice. Lessons from plaintiff depositions. Arch Intern Med 1994; 154(12):1365-70.

9. Coulter A. Patients' view of the good doctor. Br Med J 2002; 325: 668-9.

10. Deber RB, Kraetschmer N, Irvine J. What role do patients wish to play in treatment decision-making? Arch Intern Med 1996; 156(13):1414-20.

11. Like R, Zyzanski SJ. Patient satisfaction with the clinical encounter: Social psychological determinants. Soc Sci Med 1987; 24: 351-7.

12. Wooly FR, Kane RL, Hughes CC, Wright DD. The effect of doctor patient communication on satisfaction and outcome of care. Soc Sci Med 1978; 12:123-8.

13. Sullivan M. The new subjective medicine: taking the patient's point of view on health care and health. Soc Sci Med 2003; 56(7):1595-604.
14. Nascimento, CR. O futuro cuidador: perspectives e dilemas. Bioética 2006; 14 (2):153-7.

15. Ayres JRCM. Sujeito, intersubjetividade e práticas de saúde. Ciên Saude Colet 2001; 6(1):63-72.

16. Merhy E. Um ensaio sobre o médico e suas valises tecnológicas. Interface 2000; 6:109-16.

17. Bueno LSB, Teruya KM. Momento do Pediatra com a mãe. Aleitamento materno. São Paulo: Atheneu; 2006.

18. Ong LML, de Haes JCJM, Hoos AM, Lammes FB. Doctor-patient communication: a review of the literature. Soc Sci Med 1995; 40(7):903-18.

19. Universidade Estadual de Londrina. Colegiado do Curso de Medicina. Projeto Político-Pedagógico do Curso de Medicina. Londrina; 2004.

20. Universidade Estadual de Londrina. Colegiado do Curso de Medicina. Atos Legais de Consolidação do Currículo Integrado. Londrina; 2006.

21. Epstein R, Humbert E. Defining and assessing professional competence. JAMA 2002; 287(9):226-35.

22. Silva MJP. Comunicação tem remédio - A comunicação nas relações interpessoais em saúde. 2. ed. São Paulo: Gente; 1996.

23. Nunes SOV et al. O Ensino da Psiquiatria na Graduação de Medicina pelo Método de Aprendizagem Baseada em Problemas (PBL). Psiquiatr Biol 2002;10(1):23-6.

24. Thomson Z, Silva SS. Manual de Habilidades. Londrina: Universidade Estadual de Londrina; 1998.

25. World Psychiatric Association. IGDA 2: interviewing the patient. Br J Psychiatry 2003; 182 (Suppl 45): s42-s3.

\section{CONFLITOS DE INTERESSE}

Declarou não haver.

\section{ENDEREÇO PARA CORRESPONDÊNCIA}

Barbara Turini

Av. Robert Koch, 60

86038-440 - Londrina - PR

marctavares@uol.com.br ; turini@sercomtel.com.br 